\title{
CLINICAL AND ULTRASOUND PARALLELS IN DIAGNOSIS OF GASTROESOPHAGEAL REFLUX DISEASE AND AUTOIMMUNE THYROIDITIS
}

\author{
Aleksey Oparin \\ Department of Therapy, Rheumatology and Clinical Pharmacology ${ }^{1}$ \\ Natalia Yarantseva \\ Department of Therapy, Rheumatology and Clinical Pharmacology ${ }^{1}$ \\ ya.nata0505@gmail.com \\ Anatoliy Oparin \\ Department of Therapy, Rheumatology and Clinical Pharmacology ${ }^{1}$ \\ ${ }^{1}$ Kharkiv Medical Academy of Postgraduate Education \\ 58 Amosova str., Kharkiv, Ukraine, 61176
}

\begin{abstract}
The aim of the work: to study the relationship of ultrasound parameters of the thyroid gland with the clinical course, the level of thyroid hormones and indicators of the motor-secretory function of the gastrointestinal tract in patients with gastroesophageal reflux disease (GERD) and autoimmune thyroiditis (AIT).

Materials and methods. The study involved three groups of patients. The first group consisted of 22 patients with isolated GERD. The second group included 20 patients suffering from GERD in combination with AIT in the hypothyroid stage. And the third group included 20 patients suffering from isolated AIT in the hypothyroid stage. The control group consisted of 15 apparently healthy individuals of the same age and sex.

All patients underwent a comprehensive examination, which included the necessary set of clinical, laboratory and instrumental research methods in accordance with the local treatment protocols such as collection of complaints and anamnesis, patient examination, physical examination, clinical and biochemical analyzes, ECG, EGD, intragastric pH-metry. The patients underwent ultrasound examination of the motor-evacuation function of the esophagus and stomach with water load and an ultrasound examination of thyroid gland. The functional activity of thyroid gland was assessed by determining the level of thyroid hormones (TSH, T4 free, T3) by ELISA and the presence of antibodies to.

Results. The study involved three groups of patients: patients with isolated gastroesophageal reflux disease, patients with a combined course of GERD and AIT in the hypothyroid stage and patients with isolated AIT in the hypothyroid stage. The most pronounced complaints, a higher level of thyroid-stimulating hormone and the most pronounced ultrasound changes are noted in patients with combined pathology. Ultrasound examination of the lower third of esophagus and stomach in patients of all three groups showed the presence of regurgitation, expansion of the lumen of the lower third of esophagus and an increase in the diameter of esophageal opening of diaphragm in varying degrees, in contrast to patients in the control group. Thus according to the results of thyroid sonography and ultrasound examination of the motor function of the lower third of esophagus and stomach, the most pronounced changes were found in the second group of patients who had a combined course of GERD and AIT.

Conclusions. 1. A clear correlation was established between the clinical course, an increase in the level of thyroid hormones and ultrasound picture of thyroid gland and indicators of the motor-secretory function of the gastrointestinal tract in patients with GERD and AIT;

2. It was shown that disorders in the activity of thyroid hormones affect the formation of motor-secretory disorders in patients with GERD with concomitant AIT which mutually aggravates the course of this comorbid pathology;

3. Ultrasound diagnostics can be used as an evaluative method in this category of patients.

Keywords: gastroesophageal reflux disease, autoimmune thyroiditis, thyroid ultrasound, thyroid hormones.
\end{abstract}

DOI: $10.21303 / 2504-5679.2020 .001520$

\section{Introduction}

There has been a trend in recent decades towards an increase in the incidence of gastroesophageal reflux disease (GERD) especially among young people [1]. GERD symptoms occur in almost half of the adult population [2]. The leading symptom of GERD - recurrent heartburn which 
significantly reduces the quality of life - is noted by up to $40 \%$ of residents of industrialized countries. However the true prevalence of GERD in the population has not been sufficiently studied which is due to low demand for medical care, frequent asymptomatic course, as well as the course of the disease under the masks of the so-called «extraesophageal» manifestations [3].

At the same time the number of GERD patients with concomitant pathology is increasing. One of these diseases is autoimmune thyroiditis (AIT). AIT is one of the most common thyroid diseases [4]. Both of these diseases have several common pathogenetic mechanisms, the main of which are thyroid hormones, which regulate the processes of motility and secretion of the gastrointestinal tract $[5,6]$.

An autoimmune inflammatory process in thyroid gland leads to the constant production of autoantibodies to thyroperoxidase or thyroglobulin and further leads to permanent destruction of thyroid gland with the subsequent development of hypothyroidism. Early diagnosis of AIT allows early treatment and preservation of thyroid function [7, 8]. One of the most widely used methods for the diagnosis of AIT is ultrasound, so in this article we decided to study the features of ultrasound picture of thyroid gland in patients with GERD with concomitant AIT [9, 10].

The aim of the work: to study the relationship of ultrasound parameters of thyroid gland with the clinical course, the level of thyroid hormones and indicators of the motor-secretory function of the gastrointestinal tract in patients with GERD and AIT.

\section{Materials and methods}

The clinical part of the work was carried out on the basis of the Municipal Nonprofit Enterprise of Kharkiv Town Council «City Student Hospital» (Kharkiv) during 2017-2019.

The study involved three groups of patients. The first group consisted of 22 patients with isolated GERD (13 women and 9 men, average age 20.5 \pm 1.8 years). The second group included 20 patients suffering from GERD in combination with AIT in the hypothyroid stage (12 women and 8 men, average age $21.2 \pm 1.7$ years). And the third group included 20 patients suffering from isolated AIT in the hypothyroid stage (11 women and 9 men, average age $21.0 \pm 1.5$ years). The control group consisted of 15 apparently healthy individuals of the same age and sex.

During the study the principles of the Declaration of Helsinki adopted by the General Assembly of the World Medical Association (1964-2000), the Council of Europe Convention on Human Rights and Biomedicine (1997), the relevant provisions of the WHO, the International Council of Medical Scientific Communities, the International Code of Medical ethics (1983) and the laws of Ukraine were followed. It was approved by ethics committee at Kharkiv Medical Academy of Postgraduate Education (protocol No. 8 of 27.09.2017). All patients voluntarily signed an informed consent before starting the study

GERD was diagnosed based on ICD-10. All patients underwent a comprehensive examination, which included the necessary set of clinical, laboratory and instrumental research methods in accordance with the local treatment protocols such as collection of complaints and anamnesis, patient examination, physical examination, clinical and biochemical analyzes, ECG, EGD, intragastric pH-metry.

The patients underwent ultrasound examination of the motor-evacuation function of the esophagus and stomach with water load using the ULTIMA pro-30 apparatus (Ukraine). Before the study the patient had drank 1.0-1.5 liters of water. The thickness of the esophageal wall, the diameter of the esophageal opening of the diaphragm, and the diameter of the esophagus in the lower third were measured 3, 5, 10, and 15 minutes after fluid intake and the presence, and duration of gastroesophageal reflux was also established [11, 12].

The diagnosis of AIT was established on the basis of ICD 10 and on the materials of clinical protocols and clinical guidelines of the American Thyroid Association for the diagnosis and treatment of hypothyroidism $[13,14]$. An increase in antibodies to thyroid peroxidase (TPO), an increase in thyroid stimulating hormone (TSH) and changes in the ultrasound picture of the thyroid gland were taken into account. The functional activity of thyroid gland was assessed by determining the level of thyroid hormones (TSH, T4 free, T3) by ELISA and the presence of antibodies to TPO using a set of standard reagents from the «Vector-Best» company (Russia). The patients underwent an ultrasound examination of thyroid gland using the ULTIMA pro-30 apparatus (Ukraine) [15-17]. 
The data were statistically processed by using Microsoft Excel 2007 and Windows Statistika 6.0. For all types of analysis differences were considered statistically significant at $p<0.05$. Under normal distribution, Student's t-test was used; in case of deviation from the normal distribution, nonparametric methods were used. The mean value $(M)$, Student's t-test was assessed. The differences were considered significant at $p<0.05$. The results were presented as $M \pm m$, where $M$ is the median and $\mathrm{m}$ is the standard deviation.

\section{Results}

On admission patients with GERD complained of heartburn for a long time, recurrent epigastric pain, heaviness in the abdomen, belching, sour taste in the mouth, occasionally nausea, chest pain. Most of the patients noted the appearance of these complaints after dietary disturbances and/or stressful situations. Among patients of the second group along with standard dyspeptic complaints, constipation, drowsiness, mood swings, lethargy, apathy, increased anxiety about their own health were noted. Patients of the third group presented minimal complaints, more often in the form of increased fatigue, drowsiness, deterioration of the hair condition, dry skin.

Among patients of the first group with isolated GERD, thyroid hormones and antibodies to TPO were within the normal range: TSH $0.3-4.0 \mathrm{mIU} / \mathrm{L}$, antibodies to TPO up to $35 \mathrm{IU} / \mathrm{L}$. In the second and third groups antibodies to TPO exceeded the norm by several times. In the second group increase in TSH was on average up to $11.0 \pm 1.2 \mathrm{mIU} / \mathrm{L}$, in the third $-7.8 \pm 1.5 \mathrm{mIU} / \mathrm{L}$.

Ultrasound examination of the thyroid gland of patients of the first group with isolated GERD showed that the structure of the gland was normal.

In the study of patients of the second group with combined pathology the following data were obtained:

$-90 \%$ had a heterogeneous structure of the thyroid gland due to fibrous inclusions as well as a seal of the capsule;

$-9 \%$ of patients had single enlarged follicles in the structure of the thyroid gland;

- and in $3.5 \%$ of cases - areas of reduced echogenicity;

- an increase in the size of the thyroid gland was observed in $2 \%$ of patients.

Among patients of the third group with isolated AIT in $75 \%$ a heterogeneous structure of the thyroid gland was revealed due to fibrous inclusions and in $30 \%$ of patients the capsule was compacted [10].

Ultrasound examination of the lower third of the esophagus and stomach among patients of all three groups to one degree or another showed the presence of regurgitation, expansion of the lumen of the lower third of the esophagus and an increase in the diameter of the esophageal opening of the diaphragm in contrast to patients in the control group (Table 1).

Table 1

Functional state of the lower third of the esophagus during ultrasound examination with water load

\begin{tabular}{lcccc}
\hline \multicolumn{1}{c}{ Indicator } & Group 1 & Group 2 & Group 3 & Control group \\
\hline Regurgitation & + & + & + & - \\
The width of the lumen of the lower third of the esophagus & $29.3 \pm 0.7 \mathrm{~mm}$ & $32.1 \pm 0.5 \mathrm{~mm}$ & $25.7 \pm 0.6 \mathrm{~mm}$ & $22.8 \pm 0.3 \mathrm{~mm}$ \\
The diameter of the hiatal & $17.8 \pm 0.6 \mathrm{~mm}$ & $19.2 \pm 0.8 \mathrm{~mm}$ & $16.9 \pm 0.5 \mathrm{~mm}$ & $14.2 \pm 1.3 \mathrm{~mm}$
\end{tabular}

\section{Discussion}

As we can see the most expressed complaints and a higher level of TSH were among patients with combined pathology.

Thus according to the results of sonography of the thyroid gland and ultrasound examination of the motor function of the lower third of the esophagus and stomach of the second, pronounced changes were found in patients of the group who have a combined course of GERD and AIT.

Also would be desirable to draw attention to the fact that according to the results of ultrasound examination of the lower third of the stomach, signs of regurgitation and failure of the lower esophageal sphincter were noted in all three groups, including the third group with isolated AIT. 
Disruption of the gastrointestinal tract in patients with AIT was caused by the influence of thyroid hormones. With a decrease in thyroid hormones in hypothyroidism, the level of mucinous edema of the gastrointestinal tract mucosa, the secretion of gastric juice decreases, peristalsis and food passage slow down, the muscle tone of the stomach and the lower esophageal sphincter decreases, which causes the occurrence of GERD [4, 9].

The obtained data are new, since the previous studies touched upon certain aspects of the topic we are researching.

So, M. E. Solodenova et al. studied the features of the level of thyroxine, triiodothyronine, TSH and the titer of antibodies to thyroglobulin in patients with GERD with concomitant AIT. It was found that a decrease in the level of iodothyronines indicated a disruption in the work of the NPS in case of hypofunction of the thyroid gland. The possibility of a direct damaging effect on the smooth muscles of the esophageal sphincter apparatus of autoantibodies to thyroglobulin, the titer of which was increased in all patients with GERD and increased depending on the stage of the disease, was shown. However, the study did not study the features of esophageal and gastric motility and secretion depending on the level of thyroid hormones [18].

In the work of I. V. Baranov and T. V. Maikova, the features of the content of titers of antibodies to thyroperoxidase and thyroglobulin were studied and the state of morphology of the esophageal mucosa was analyzed. A clear relationship was revealed between the level of thyroid hormones and the severity of atrophy and inflammatory changes in the mucous membrane of the esophagus. However, the authors did not investigate the secretion features in this group of patients, as well as the clinical course of GERD, depending on the presence of AIT [19].

In the work of V. A. Akhmedov, the possibilities of conservative therapy in patients with GERD are analyzed, taking into account the characteristics of secretion, indicators of psychosomatics and quality of life. However, the features of therapy with proton pump inhibitors in patients with concomitant pathology affecting the state of secretion, in particular in the presence of AIT, and the role of thyroid hormones have not been clarified [20].

In the work of V. T. Ivashkin and I. V. Maev, the features of GERD therapy were analyzed in detail, taking into account the clinical picture of the disease, the characteristics of secretion and the morphological state of the GERD mucosa. However, the features of the course of GERD in the presence of concomitant pathology and the effect of thyroid hormones on the course of GERD and the formation of motor-secretory disorders have not been studied [3].

Thus, our study, on the one hand, supplements the information about the features of the clinical course of GERD with concomitant AIT, and on the other hand, contributes to the disclosure of the mechanisms of the formation of motor secretory disorders in patients with GERD with concomitant AIT, which will make it possible to develop a differentiated therapy for this comorbid pathology.

Study limitations. The study was not conducted in case of identified concomitant pathology of other organs and in case of patients refuse to participate in the study.

Prospects for further research. Further study of the effect of thyroid hormones on the clinical course of isolated GERD and GERD with combined pathology is important for the formation of treatment and prevention regimens for a particular patient, and at the same time it outlines new directions in approaches to both diagnosis and treatment of this pathology.

\section{Conclusions}

1. A clear correlation has been established between the clinical course, the increase in the level of thyroid hormones and the ultrasound picture of the thyroid gland and indicators of the motorsecretory function of the gastrointestinal tract in patients with GERD and AIT.

2. It was shown that disorders in the activity of thyroid hormones affect the formation of motorsecretory disorders in patients with GERD with concomitant AIT which mutually aggravates the course of this comorbid pathology.

3. Ultrasound diagnostics can be used as an evaluative method in this category of patients.

\section{Conflict of interests}

The authors declare that they have no conflicts of interest. 


\section{References}

[1] Pandit, S., Boktor, M., Alexander, J. S., Becker, F., Morris, J. (2018). Gastroesophageal reflux disease: A clinical overview for primary care physicians. Pathophysiology, 25 (1), 1-11. doi: http://doi.org/10.1016/j.pathophys.2017.09.001

[2] Starostin, B. D. (2014). Gastroesophageal reflux disease (part I). Epidemiology, risk factors. Gastroenterology of St. Petersburg, 1-2, 2-14.

[3] Ivashkin, V. T., Maev, I. V., Trukhmanov, A. S. et. al. (2014) Gastroesophageal reflux disease. Clinical recommendations. Moscow: Russian Gastroenterological Association, 23.

[4] Goncharova, O. A. (2020). Associative relations between hypofunction of the thyroid gland of various origins and iron deficiency anemia. Reproductive Endocrinology, 51, 60-62. doi: http://doi.org/10.18370/2309-4117.2020.51.60-62

[5] Cellini, M., Santaguida, M. G., Virili, C., Capriello, S., Brusca, N., Gargano, L., Centanni, M. (2017). Hashimoto's Thyroiditis and Autoimmune Gastritis. Frontiers in Endocrinology, 8. doi: http://doi.org/10.3389/fendo.2017.00092

[6] Antonelli, A., Benvenga, S. (2018). Editorial: The Association of Other Autoimmune Diseases in Patients With Thyroid Autoimmunity. Frontiers in Endocrinology, 9. doi: http://doi.org/10.3389/fendo.2018.00540

[7] Smirnov, V. V., Ushakov, A. I. (2020). Timely diagnostics and treatment of hypothyroidism is a basis for normal physical and psychical development of children (part 1). Attending physician, 1, 10-16.

[8] Smirnov, V. V., Makazan, N. V. (2010) Thyrotoxicosis syndrome: causes, diagnosis, treatment. Attending physician, 5.

[9] Sagitova, G. R., Otto, N. Yu. (2011). Echographic characteristics of thyroid lesions in children of the Astrakhan region. Attending physician, 11.

[10] Tukhbatullin, M. G. (2016). Echography in the diagnosis of diseases of internal and superficial organs. Kazan: Meditsinskaya kniga, 208.

[11] Yusupova, R. A., Podolskaya, T. B. (2017). Ultrasonic investigation of the autoimmune thyroiditis in out-patient conditions. Practical Medicine 2 (17).

[12] Oparin, A. A., Oparin, A. G., Korenovsky, I. P., Lavrova, N. V.; Oparin, A. A. (Ed.) (2012). Algorithms and criteria for ultrasound diagnostics in the clinic of internal diseases. Kharkiv: Fact, 80.

[13] Oparin, A., Kornienko, D. (2017). Formation process of motor-evacuatory disorders in patients with gastroesophageal reflux disease and concomitant obesity. Gastroenterologie a Hepatologie, 71 (2), 145-149. doi: http://doi.org/10.14735/amgh2017csgh.info01

[14] Jonklaas, J., Bianco, A. C., Bauer, A. J., Burman, K. D., Cappola, A. R., Celi, F. S. et. al. (2014). Guidelines for the Treatment of Hypothyroidism: Prepared by the American Thyroid Association Task Force on Thyroid Hormone Replacement. Thyroid, 24 (12), 1670-1751. doi: http://doi.org/10.1089/thy.2014.0028

[15] Balagansky, D. A., Kirgizov, I. V., Koshel, A. P., Vrublevskaya, M. L. (2013). Transabdominal ultrasonography in the diagnosis of gastroesophageal reflux in young children. Pediatric surgery, 2, 14-18.

[16] Cherniavskaya, I. V., Zemlianitsyna, O. V., Cherniaieva, A. A., Dunaeva, I. P., Oleynikova, S. P., Kravchun, N. A. (2018). The place of phytotherapy in complex treatment of autoimmune diseases of the thyroid gland. Endocrine pathology problems, 1 , 62-70. doi: http://doi.org/10.21856/j-pep.2018.1.08

[17] Vagapova, G. R., Khamzina, F. T. (2008). Application of ultrasound examination of the thyroid gland in the color Doppler mode for the diagnosis of autoimmune thyroiditis. Practical Medicine, 3 (27), 33-35.

[18] Solodenova, M. E., Luzina, E. V., Suvorova, T. V. (2008). Features of the thyroid gland function in patients with gastroesophageal reflux disease. Far Eastern medical journal, 21-24.

[19] Baranov, I. V., Maikova, T. V. (2010). Endoscopic characteristics of the esophagus, stomach and duodenum in patients with gastroesophageal reflux disease in combination with primary hypothyroidism. News of Medicine and Pharmacy. Gastroenterology, 337.

[20] Ametov, A. S. (2012). Selected lectures on endocrinology. Moscow: Medical information agency, 544. 